\title{
A note on multi-objective integer linear programming problems using pentagonal fuzzy numbers
}

\author{
D. Stephen Dinagar ${ }^{1 *}$ and M. Mohamed Jeyavuthin ${ }^{2}$
}

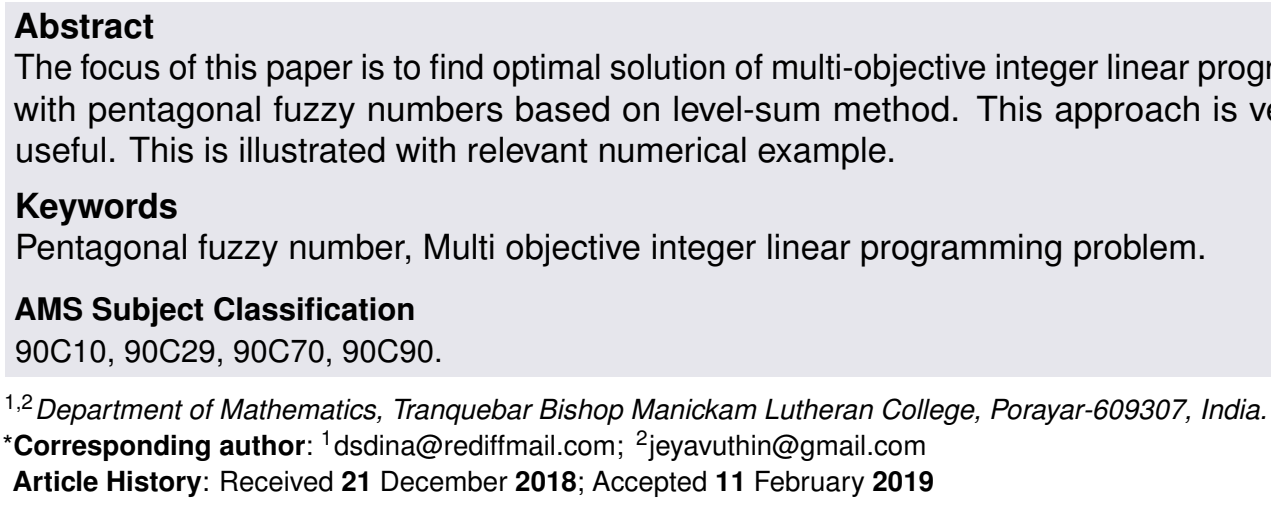

\section{Contents}

1 Introduction $\ldots \ldots \ldots \ldots \ldots \ldots \ldots \ldots \ldots \ldots \ldots \ldots \ldots, 48$

2 Preliminaries $\ldots \ldots \ldots \ldots \ldots \ldots \ldots \ldots \ldots \ldots \ldots \ldots, 48$

3 Fuzzy Integer Linear Programming Problem..... . .49

4 Numerical Example.........................50

5 Conclusion ........................... 51

References ............................. 51

\section{Introduction}

The concept of linear programming problem is to find out the best solution to the real-world problems where the available information's are not exact or not precise. In that situation linear programming model can be applied. It plays a vital role in fuzzy modeling, which can formulate the uncertainty.

In [2] Herrera and Verdegay studied some models for dealing with fuzzy integer linear programming problems by using fuzzy constraints and fuzzy numbers. Allahviranloo et al.[1] have introduced a new method by reducing the fuzzy integer linear programming models to crisp integer linear programming models. Pandian [3] solved fuzzy linear programming problems using multi-objective technique. Sahaya sudha et al. [4] discussed multi-objective fuzzy linear programming problem with pentagonal fuzzy numbers. In [5] Stephen Dinagar and Mohamed Jeyavuthin discussed the concept of solving integer linear programming problems with pentagonal fuzzy numbers.
In this paper, section 2 contains some basic definitions needed for this work. In section 3, fuzzy integer linear programming with multi-objective functions are discussed. In section 4, A relevant numerical illustration is given. Finally, conclusion is included in section 5 .

\section{Preliminaries}

In this section, we recall some definitions and basic results which will be used throughout the paper.

Definition 2.1. [5] A fuzzy set $\tilde{A}$ is defined by

$$
\tilde{A}=\left\{\left(x, \mu_{A}(x)\right): x \in A, \mu_{A}(x) \in[0,1]\right\} .
$$

In the pair $\left(x, \mu_{A}(x)\right)$, the first element $x$ belong to the classical set $A$, the second element $\mu_{A}(x)$, belong to the interval $[0,1]$, called Membership function.

Definition 2.2. [5] A fuzzy set $\tilde{A}$ is convex if

$$
\mu_{\tilde{A}}\left(\lambda x_{1}+\left(1-\lambda x_{2}\right) \geq \min \left(\mu_{\tilde{A}}\left(x_{1}\right), \mu_{\tilde{A}}\left(x_{2}\right)\right)\right), x_{1}, x_{2} \in X
$$

and $\lambda \in[0,1]$. Alternatively, a fuzzy set is convex, if all $\alpha$-level sets are convex.

Definition 2.3. [5] A fuzzy set $\tilde{A}$ is defined on the set of real numbers $R$ is said to be fuzzy number if it has the following characteristics

(i) $\tilde{A}$ is normal

(ii) $\tilde{A}$ is convex set

(iii) The support of $\tilde{A}$ is closed and bounded. 
Definition 2.4. [5] A fuzzy number $\tilde{A}_{P}$ is Pentagonal fuzzy number denoted by $\tilde{A}_{P}=\left(a_{1}, a_{2}, a_{3}, a_{4}, a_{5}\right)$, where $a_{1}, a_{2}, a_{3}, a_{4}, a_{5}$ are real numbers and its membership $\mu_{\tilde{A}_{P}}(x)$ is given by

$$
\mu_{\tilde{A}_{P}}(x)=\left\{\begin{array}{cc}
0 & , x<a_{1} \\
\frac{1}{2}\left(\frac{x-a_{1}}{a_{2}-a_{1}}\right) & , a_{1} \leq x \leq a_{2} \\
\frac{1}{2}+\frac{1}{2}\left(\frac{x-a_{2}}{a_{3}-a_{2}}\right) & , a_{2} \leq x \leq a_{3} \\
1 & , x=a_{3} \\
\frac{1}{2}+\frac{1}{2}\left(\frac{a_{4}-x}{a_{4}-a_{3}}\right) & , a_{3} \leq x \leq a_{4} \\
\left(\frac{a_{5}-x}{a_{5}-a_{4}}\right) & , a_{4} \leq x \leq a_{5} \\
0 & , x>a_{5}
\end{array}\right.
$$

Definition 2.5. A pentagonal fuzzy number can be defined as $\tilde{A}_{P}=\left(M_{1}(u), J_{1}(v), J_{2}(v), M_{2}(u)\right)$ for $u \in[0,0.5]$ and $v \in$ $[0.5,1]$

(i) $M_{1}(u)$ is strictly a increasing continuous function on [0,0.5] (ii) $J_{1}(v)$ is strictly a increasing continuous function on [0.5,1] (iii) $J_{2}(v)$ is strictly a decreasing continuous function on [1,0.5] (iv) $M_{2}(u)$ is strictly a decreasing continuous function on [0.5,1].

Remark 2.6. The pentagonal fuzzy number $\tilde{A}_{P}$ becomes triangular fuzzy number if $a_{3}-a_{2}=a_{4}-a_{3}$.

\section{Fuzzy Integer Linear Programming Problem}

Consider the following fully fuzzy integer linear programming problems:

(P) Maximize (or) Minimize $\tilde{z}=\tilde{c}^{T}$ subject to $\tilde{A} \times \tilde{x}\{\leq,=, \geq\} \tilde{b}, \tilde{x} \geq 0$ and are integers,

where the cost vectors $\tilde{c}^{T}=\left(\tilde{c}_{j}\right)_{1 \times n}, \tilde{A}=\left(\tilde{a}_{i j}\right)_{m \times n}, \tilde{x}=\left(\tilde{x}_{j}\right)_{n \times 1}, \tilde{b}=\left(\tilde{b}_{i}\right)_{m \times 1}$ and $\tilde{a}_{i j}, \tilde{x}_{j}, \tilde{b}_{i}, \tilde{c}_{j} \in F(R)$, for all $1 \leq j \leq n$ and $1 \leq i \leq m$.

Let the parameters $\tilde{z}_{j}, \tilde{a}_{i j}, \tilde{x}_{j}, \tilde{b}_{i}, \tilde{c}_{j}$ be the pentagonal fuzzy numbers

$$
\begin{array}{rlrl}
\tilde{z} & =\left(z_{1}, z_{2}, z_{3}, z_{4}, z_{5}\right), & \tilde{x}_{j}=\left(x_{j}, y_{j}, t_{j}, u_{j}, v_{j}\right) \\
\tilde{a}_{i j} & =\left(a_{i j}, b_{i j}, c_{i j}, d_{i j}, e_{i j}\right), & \tilde{b}_{i}=\left(b_{i}, e_{i}, f_{i}, g_{i}, h_{i}\right) \\
\tilde{c}_{j} & =\left(p_{j}, q_{j}, r_{j}, s_{j}, t_{j}\right) & &
\end{array}
$$

respectively.

The mathematical formulation as follows:

$$
\begin{aligned}
(P) \operatorname{Maximize} & \left(z_{1}, z_{2}, z_{3}, z_{4}, z_{5}\right) \\
= & \sum_{j=1}^{n}\left(p_{j}, q_{j}, r_{j}, s_{j}, t_{j}\right) \times\left(x_{j}, y_{j}, t_{j}, u_{j}, v_{j}\right)
\end{aligned}
$$

subject to

$$
\begin{aligned}
& \sum_{j=1}^{n}\left(p_{j}, q_{j}, r_{j}, s_{j}, t_{j}\right) \times\left(x_{j}, y_{j}, t_{j}, u_{j}, v_{j}\right) \\
&\{\leq,=, \geq\}\left(b_{i}, e_{i}, f_{i}, g_{i}, h_{i}\right) \\
&\left(x_{j}, y_{j}, t_{j}, u_{j}, v_{j}\right) \geq 0,(j=1,2,3, \ldots, m)
\end{aligned}
$$

and are integers.

$a_{5}$ Now, we have to transform the fuzzy integer linear programming problem into multi-objective fuzzy integer linear programming problem by using level-sum method.

$$
\begin{aligned}
& \begin{array}{r}
\text { M) Maximize } z_{1} \\
=
\end{array} \sum_{j=1}^{n} \text { most lowest value of } \\
&\left(p_{j}, q_{j}, r_{j}, s_{j}, t_{j}\right) \times\left(x_{j}, y_{j}, t_{j}, u_{j}, v_{j}\right) \sum_{j=1}^{n} \text { lowest value of } \\
& \text { Maximize } z_{2}=\left(p_{j}, q_{j}, r_{j}, s_{j}, t_{j}\right) \times\left(x_{j}, y_{j}, t_{j}, u_{j}, v_{j}\right) \\
& \text { Maximize } z_{3}=\sum_{j=1}^{n} \text { middle value of } \\
&\left(p_{j}, q_{j}, r_{j}, s_{j}, t_{j}\right) \times\left(x_{j}, y_{j}, t_{j}, u_{j}, v_{j}\right) \\
& \text { Maximize } z_{4}=\sum_{j=1}^{n} \text { upper value of } \\
&\left(p_{j}, q_{j}, r_{j}, s_{j}, t_{j}\right) \times\left(x_{j}, y_{j}, t_{j}, u_{j}, v_{j}\right) \\
& \text { Maximize } z_{5}=\sum_{j=1}^{n} \text { most upper value of } \\
&\left(p_{j}, q_{j}, r_{j}, s_{j}, t_{j}\right) \times\left(x_{j}, y_{j}, t_{j}, u_{j}, v_{j}\right)
\end{aligned}
$$

subject to constraints

$$
\begin{aligned}
& \sum_{j=1}^{n} \text { most lowest value of } \\
& \qquad\left(p_{j}, q_{j}, r_{j}, s_{j}, t_{j}\right) \times\left(x_{j}, y_{j}, t_{j}, u_{j}, v_{j}\right)\{\leq,=, \geq\} b_{i}
\end{aligned}
$$$$
\sum_{j=1}^{n} \text { lowest value of }
$$$$
\left(p_{j}, q_{j}, r_{j}, s_{j}, t_{j}\right) \times\left(x_{j}, y_{j}, t_{j}, u_{j}, v_{j}\right)\{\leq,=, \geq\} e_{i}
$$

$\sum_{j=1}^{n}$ middle value of

$$
\left(p_{j}, q_{j}, r_{j}, s_{j}, t_{j}\right) \times\left(x_{j}, y_{j}, t_{j}, u_{j}, v_{j}\right)\{\leq,=, \geq\} f_{i}
$$

$\sum_{j=1}^{n}$ upper value of

$$
\left(p_{j}, q_{j}, r_{j}, s_{j}, t_{j}\right) \times\left(x_{j}, y_{j}, t_{j}, u_{j}, v_{j}\right)\{\leq,=, \geq\} g_{i}
$$

$\sum_{j=1}^{n}$ most upper value of

$$
\left(p_{j}, q_{j}, r_{j}, s_{j}, t_{j}\right) \times\left(x_{j}, y_{j}, t_{j}, u_{j}, v_{j}\right)\{\leq,=, \geq\} h_{i}
$$

for all $i=1,2,3, \ldots, m$

$$
\begin{aligned}
& z_{2} \geq z_{1} ; z_{3} \geq z_{2} ; z_{4} \geq z_{3} ; z_{5} \geq z_{4} \\
& x_{j} \leq y_{j}, j=1,2,3, \ldots, m, y_{j} \leq t_{j}, j=1,2,3, \ldots, m, \\
& t_{j} \leq u_{j}, j=1,2,3, \ldots, m, u_{j} \leq v_{j}, j=1,2,3, \ldots, m, \\
& x_{j} \geq 0, j=1,2,3, \ldots, m .
\end{aligned}
$$

Note 3.1. Suppose a fuzzy integer linear programming problem having hexagonal fuzzy number is considered, we will 
have a multi-objective linear programming problem with six objectives. Here we establish the following theorem which gives relation between optimal solution of the fuzzy integer linear programming problem and an efficient solution to the corresponding multi-objective problem.

Definition 3.2. [4]

Let $\tilde{A}_{P}=\left(a_{1}, a_{2}, a_{3}, a_{4}, a_{5}\right)$ and $\tilde{B}_{P}=\left(b_{1}, b_{2}, b_{3}, b_{4}, b_{5}\right)$ be in $F(R)$ then $\tilde{A}>\tilde{B}$ if and only if $a_{i} \geq b_{i}, j=1$ to 5 and $b_{r}>a_{r}$ for some $r \in\{1,2,3,4,5\}$.

Definition 3.3. [3]

A feasible point $x^{0}$ is said to be efficient solution if there exists no other feasible point $x$ in $P$ such that $f_{i}(x) \leq f_{i}\left(x^{0}\right), i=$ $1,2, \ldots, k$ and $f_{r}(x)<f_{r}\left(x^{0}\right)$ for some $r \in\{1,2, \ldots, k\}$.

Theorem 3.4. Let $X^{0}=\left\{x_{j}^{0}, y_{j}^{0}, t_{j}^{0}, u_{j}^{0}, v_{j}^{0} ; j=1,2, \ldots, m\right\}$ be an efficient solution to the problem (M).Then,

$\tilde{X}^{0}=\left\{\left(x_{j}^{0}, y_{j}^{0}, t_{j}^{0}, u_{j}^{0}, v_{j}^{0}\right) ; j=1,2, \ldots, m\right\}$ is an optimal solution to the problem $(P)$.

Proof. Let $X^{0}=\left\{x_{j}^{0}, y_{j}^{0}, t_{j}^{0}, u_{j}^{0}, v_{j}^{0} ; j=1,2, \ldots, m\right\}$ be an efficient solution to the problem (M).Then,

$\tilde{X}^{0}=\left\{\left(x_{j}^{0}, y_{j}^{0}, t_{j}^{0}, u_{j}^{0}, v_{j}^{0}\right) ; j=1,2, \ldots, m\right\}$ is a feasible solution to the problem $(\mathrm{P})$.

Assume that $\tilde{X}^{0}=\left\{\left(x_{j}^{0}, y_{j}^{0}, t_{j}^{0}, u_{j}^{0}, v_{j}^{0}\right) ; j=1,2, \ldots, m\right\}$ is not an optimal solution to the problem $(\mathrm{P})$. Then, there exists a feasible solution, $\tilde{X}^{0}=\left\{\left(x_{j}^{0}, y_{j}^{0}, t_{j}^{0}, u_{j}^{0}, v_{j}^{0}\right) ; j=1,2, \ldots, m\right\}$ to the problem $(\mathrm{P})$ such that $Z(\tilde{X})>Z(\tilde{X})^{0}$,that is, $z_{i}(x, y, t, u, v) \geq z_{i}\left(x^{0}, y^{0}, t^{0}, u^{0}, v^{0}\right), i=1,2,3,4,5$ and $z_{r}(x, y, t, u, v)>z_{r}\left(x^{0}, y^{0}, t^{0}, u^{0}, v^{0}\right)$, for some $r \in\{1,2,3,4,5\}$ where,

$$
\begin{array}{rlrl}
x^{0} & =\left\{x_{j}^{0} ; j=1,2,3, \ldots, m\right\}, & y^{0} & =\left\{y_{j}^{0} ; j=1,2,3, \ldots, m\right\}, \\
t^{0} & =\left\{t_{j}^{0} ; j=1,2,3, \ldots, m\right\}, & u^{0}=\left\{u_{j}^{0} ; j=1,2,3, \ldots, m\right\}, \\
v^{0} & =\left\{v_{j}^{0} ; j=1,2,3, \ldots, m\right\}, & x & =\left\{x_{j} ; j=1,2,3, \ldots, m\right\}, \\
y & =\left\{y_{j} ; j=1,2,3, \ldots, m\right\}, & t & =\left\{t_{j} ; j=1,2,3, \ldots, m\right\}, \\
u & =\left\{u_{j} ; j=1,2,3, \ldots, m\right\}, & v & =\left\{v_{j} ; j=1,2,3, \ldots, m\right\} .
\end{array}
$$

This means that $X^{0}=\left\{x_{j}^{0}, y_{j}^{0}, t_{j}^{0}, u_{j}^{0}, v_{j}^{0} ; j=1,2, \ldots, m\right\}$ is not an efficient solution to the problem (M). This is a contradiction, which proves the theorem.

Algorithm 3.5. The computational procedure for finding optimal solution to the fuzzy integer linear programming problem with the aid of level-sum method is given as:

Step 1 construct the multi-objective integer linear programming problem from the given fuzzy integer linear programming problem.

Step 2 Find an efficient solution to the multi-objective integer linear programming problem obtained in step 1 using the sum of objectives method [3].
Step 3 The efficient solution obtained in step 2 to the multiobjective integer linear programming problem gives an optimal fuzzy solution to the fuzzy integer linear programming problem by the above theorem.

\section{Numerical Example}

Example 4.1. Consider the following fuzzy integer linear programming problem

$$
\text { Maximize } \tilde{z}=(1,2,3,4,5) \tilde{x}_{1}+(6,7,8,9,10) \tilde{x}_{2}
$$

Subject to constraints

$$
\begin{array}{r}
(2,4,6,8,10) \tilde{x}_{1}+(3,6,9,12,15) \tilde{x}_{2} \leq(5,10,15,20,25) ; \\
(1,2,3,4,5) \tilde{x}_{1}+(2,4,6,8,10) \tilde{x}_{2} \leq(10,20,30,40,50) ;
\end{array}
$$

Let

$\tilde{x}_{1}, \tilde{x}_{2} \geq 0$ and are integers.

$$
\begin{aligned}
\tilde{x}_{1} & =\left(x_{1}, y_{1}, t_{1}, u_{1}, v_{1}\right), \tilde{x}_{2}=\left(x_{2}, y_{2}, t_{2}, u_{2}, v_{2}\right) \text { and } \\
\tilde{z} & =\left(z_{1}, z_{2}, z_{3}, z_{4}, z_{5}\right)
\end{aligned}
$$

Now, using the step 1, the multi-objective problem related to the given problem is,

$$
\begin{aligned}
\text { (M)Maximize } z_{1} & =x_{1}+6 x_{2}, & & \text { Maximize } z_{2}=2 y_{1}+7 y_{2} \\
\text { Maximize } z_{3} & =3 t_{1}+8 t_{2}, & & \text { Maximize } z_{4}=4 u_{1}+9 u_{2}, \\
\text { Maximize } z_{5} & =5 v_{1}+10 v_{2} & &
\end{aligned}
$$

Subject to constraints

$$
\begin{aligned}
& 2 x_{1}+3 x_{2} \leq 5 ; \quad 4 y_{1}+6 y_{2} \leq 10 ; \quad 6 t_{1}+9 t_{2} \leq 15 ; \\
& 8 u_{1}+12 u_{2} \leq 20 ; \quad 10 v_{1}+15 v_{2} \leq 25 ; \quad 1 x_{1}+2 x_{2} \leq 10 ; \\
& 2 y_{1}+4 y_{2} \leq 20 ; \quad 3 t_{1}+6 t_{2} \leq 30 ; \quad 4 u_{1}+8 u_{2} \leq 40 ; \\
& 5 v_{1}+10 v_{2} \leq 50 \\
& z_{2} \geq z_{1} ; z_{3} \geq z_{2} ; z_{4} \geq z_{3} ; \quad z_{5} \geq z_{4} ; y_{1} \geq x_{1} ; t_{1} \geq y_{1} ; \\
& u_{1} \geq t_{1} ; v_{1} \geq u_{1} ; y_{2} \geq x_{2} ; \quad t_{2} \geq y_{2} ; u_{2} \geq t_{2} ; v_{2} \geq u_{2}
\end{aligned}
$$

Now, by the step 2, we consider the following linear programming problem $(S)$ related to the above multi-objective integer linear programming problem,

(S) Maximize $\tilde{z}$

$=x_{1}+6 x_{2}+2 y_{1}+7 y_{2}+3 t_{1}+8 t_{2}+4 u_{1}+9 u_{2}+5 v_{1}+10 v_{2}$ subject to

$$
\begin{array}{rrr}
2 x_{1}+3 x_{2} \leq 5 ; & 4 y_{1}+6 y_{2} \leq 10 ; & 6 t_{1}+9 t_{2} \leq 15 ; \\
8 u_{1}+12 u_{2} \leq 20 ; & 10 v_{1}+15 v_{2} \leq 25 ; \quad 1 x_{1}+2 x_{2} \leq 10 ; \\
2 y_{1}+4 y_{2} \leq 20 ; & 3 t_{1}+6 t_{2} \leq 30 ; \quad 4 u_{1}+8 u_{2} \leq 40 ; \\
5 v_{1}+10 v_{2} \leq 50 ; & \\
2 y_{1}+7 y_{2}-x_{1}-6 x_{2} \leq 0 ; & 3 t_{1}+8 t_{2}-2 y_{1}-7 y_{2} \leq 0 ; \\
4 u_{1}+9 u_{2}-3 t_{1}-8 t_{2} \leq 0 ; \quad 5 v_{1}+10 v_{2}-4 u_{1}-9 u_{2} \geq 0 ; & \\
y_{1} \geq x_{1} ; t_{1} \geq y_{1} ; \quad u_{1} \geq t_{1} ; \quad v_{1} \geq u_{1} ; \\
y_{2} \geq x_{2} ; t_{2} \geq y_{2} ; \quad u_{2} \geq t_{2} ; \quad v_{2} \geq u_{2} .
\end{array}
$$


and solve it by Gomory's fractional cut method. The optimal solution to the problem $(S)$ is

$x_{1}=1, x_{2}=1, y_{1}=1, y_{2}=1, t_{1}=1, t_{2}=1, u_{1}=1$, $u_{2}=1, v_{1}=1, v_{2}=1$ with $Z=55$,

which is the efficient solution to the problem $(M)$.

Now, by using step 3, the optimal fuzzy solution to the given problem is

$\tilde{x}_{1}=(1,1,1,1,1), \tilde{x}_{2}=(1,1,1,1,1)$ and $\tilde{z}=(7,9,11,13,15)$.

\section{Conclusion}

In this work, the notion called level-sum method to find an optimal fuzzy solution to a fuzzy integer linear programming problem using pentagonal fuzzy numbers have been proposed. The main advantage of this method is easy to solve the optimal solution for the given fuzzy integer linear programming problem and it provides an appropriate best solution to different types of linear programming models. This notion can be extended to some other optimization problems in future.

\section{References}

[1] T.Allahviranloo, KH.Shamsolkotabi, N.A.Kiani and L.Alizadeh, Fuzzy integer linear programming problems, Int. J. Contemp. Math. Sciences, 2(2007), 167-181.

[2] F. Herrera and J.L.Verdegay, Three models of fuzzy integer linear programming, European Journal of Operational Research, 83(1995), 581-593.

[3] P. Pandian, Multi-objective Programming Approach for fuzzy linear programming problems,Applied Mathematical Sciences, 7(37)(2013), 1811-1817.

[4] A. Sahaya Sudha, S.Sathya and S.Vimala virginmary, Solving a multi objective linear programming using pentagonal fuzzy numbers, International Journal of Mathematics Trends and Technology, 41(3)(2017), 12-17.

[5] D. Stephen Dinagar and M.Mohamed Jeyavuthin, A Note on Integer Linear Programming Problems with LR Pentagonal Fuzzy Numbers, Journal of Computer and Mathematical Sciences, 9(9)(2018), 1093-1100.

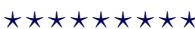

ISSN(P):2319 - 3786

Malaya Journal of Matematik

$\operatorname{ISSN}(\mathrm{O}): 2321-5666$

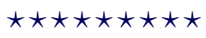

